\title{
Estudo comparativo entre as práticas empresariais e a teoria de gerenciamento por Valor Agregado: o caso da construção civil
}

\author{
Comparative study of managerial practices and EVM \\ management theory: the case of construction
}

\section{Joaquim Teixeira Netto Osvaldo Luiz Gonçalves Quelhas \\ Sergio França \\ Marcelo Jasmim Meirino}

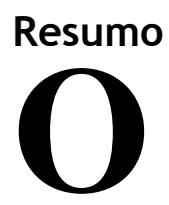

monitoramento do desempenho de projetos por meio de indicadores é um recurso de gestão de grande importância para as organizações, sendo o Earned Value Management (EVM) amplamente utilizado há aproximadamente 40 anos. Entretanto, há poucas pesquisas sobre sua utilização na indústria da construção civil. Outrossim, alguns autores argumentam quea fundamentação teórica doEVM é utilizada de forma parcial na construção civil. O presente artigo tem como objetivo principal o estudo comparativoentre a fundamentação teórica e aprática no uso do indicador de EVM. A pesquisa do tipo exploratória foi conduzida por meio do estudo de caso em 05 projetos de obras civis. A pesquisa de campo buscou identificaras práticas utilizadas, bem como compará-las com a teoria de EVM. Por fim, pôde-se concluir, com estes estudos de caso, que há fatores críticos de sucesso, tais como: o treinamento da equipe, a maturidade em gestão de projetos, o apoio da alta administração, a necessidade de procedimentos pré-definidos. Observou-se, ainda, que o EVM propiciou benefícios como um maior controle do custo, escopo e comunicação do desempenho do projeto. Finalmente, verificou-se que as empresas não utilizam a totalidade de requisitos teóricos do EVM. Ademais, constatou-se que o método utilizado na pesquisa permitiu sucesso no desenvolvimento dos objetivos e na validação das hipóteses.

Palavras-chaves: Indicador de valor agregado. EVM. Fator crítico de sucesso. Desempenho da construção civil. Gestão de projetos.

\begin{abstract}
Indicators are a major management tool that organizations use to monitor project performance. The EVM indicator has widely been used for about 40 years. Nevertheless, only few studies have been conducted about its use in the construction industry. In addition, some authors claim that the theoretical foundation of EVM has been incorrectly used in the construction industry. The main objective of this article is to present a comparative study of the theoretical foundations and the practical use of the EVM indicator. This is an exploratory study involving a case study in 5 construction projects. After the field research, the practices used were identified and compared with the EVM theory. The researchers observed that there were critical success factors such as: staff training, maturity in project management, support from high management, and the need of pre-defined procedures. They also observed that the EVM offered benefits such as better cost control, scope and communication about the project's performance. Additionally, they concluded that the method applied enabled the successful achievement of the goals and the validation of the research hypothesis.
\end{abstract}

Marcelo Jasmim Meirino Universidade Federal Fluminense Niterói - RJ Brasil

Recebido em 04/10/14 Aceito em 05/05/15
Keywords: Earned value indicator. EVM. Critical success factors. Construction performance. Project management. 


\section{Introdução}

O aumento da competição em escala global e o rápido desenvolvimento tecnológico têm motivado as empresas a melhorar seus controles internos de projetos (KIM; WELLS JUNIOR; DUFFEY, 2003). Os indicadores de desempenho de projetos são instrumentos de gestão essenciais nas atividades de monitoramento de avaliação, uma vez que permitem o acompanhamento e o alcance das metas, além da identificação de avanços e melhorias de qualidade. Monitorar o projeto significa comparar a situação atual com a planejada, verificando se seus custos e seus prazos estão dentro do planejado, adotando medidas corretivas quando for necessário (DE MARCO; NARBAEV, 2013). A indústria da construção civil apresenta enorme gasto e baixa eficiência quando comparada com outras indústrias. Esse fato ocorre em razão da deficiente utilização dos mecanismos de gestão de projetos (DOBBS et al., 2013). Assim, os índices de desempenho de projetos na área de engenharia têm especial importância, pois permitem antever problemas que poderão ocorrer durante o andamento deles, possibilitando que ajustes e correções possam ser feitos de modo a evitar desvios no planejamento.

O gerenciamento por valor agregado é uma ferramenta essencial para gerenciar o escopo, o prazo e o custo, possibilitando a obtenção do índice de desempenho do projeto (NARBAEV; DE MARCO, 2014). Atualmente, as pesquisas sobre controles de prazos são cada vez mais frequentes, apresentando-se em diversos modelos e aplicações (VANHOUCKE, 2012). Entretanto, há poucas pesquisas que abordam a gestão de projetos da construção civil com a utilização do desempenho de valor agregado.

Embora sendo notória sua utilização, a aplicação do indicador de valor agregado em projetos não é de fácil execução. Ainda assim, a indústria da construção civil, que envolve projetos complexos e com inúmeras incertezas, adotou o indicador do valor agregado para o controle de seus custos (ALJIBOURI, 2003).

Fleming e Koppelman (2010) argumentam sobre a importância da utilização de metodologias e de procedimentos adequados para monitorar o desempenho através do valor agregado. Além desses aspectos, requer treinamento prévio e disponibilidade de um sistema de informações (PRACTICE..., 2011). Segundo Kim, Wells Junior e Duffey (2003), a maior incidência de problemas identificados no uso do EVM se refere ao entendimento da metodologia e à falta da utilização de um software integrado de gestão de projetos.
A indústria da construção civil, na utilização prática do EVM, encontra-se desatualizada quando comparada a outras indústrias e apresenta dificuldades em adequar a metodologia à prática de gerenciamento de projetos (DE MARCO; NARBAEV, 2013). Segundo Fleming e Koppelman (2010), algumas empresas da construção utilizam de maneira equivocada o valor planejado de custos e o valor atual de custos, não considerando a terceira dimensão, que é o valor agregado. Lipke (2013) argumenta que a utilização do valor agregado não é simples e necessita de um gerenciamento complexo. Segundo Song (2010), o indicador de valor agregado é conhecido na indústria da construção como agregado em horas ou agregado em dólares. Segundo Kim, Wells Junior e Duffey (2003), o treinamento e a prática do EVM é um fator crítico de sucesso. Os mesmos autores afirmam que os estudos teóricos referentes ao assunto de EVM são numerosos. Entretanto, a quantidade de estudos empíricos sobre o tema é reduzida. Por isso autores Fleming e Koppelman (2010), Song (2010), Kim, Wells Junior e Duffey (2003), De Marco e Narbaev (2013) argumentam que a indústria da construção civil não utiliza totalmente a metodologia de valor agregado.

Pesquisas relativas aos fatores críticos de sucesso para o uso do EVM na construção civil foram abordadas por Buyse, Vandenbussche e Vanhoucke (2009), Vargas (2003) e Valle e Soares (2006). Além disso, pesquisas recentes sobre o EVM têm sido realizadas por Narbaev e De Marco (2014) e Vanhoucke (2012), porém nenhuma delas aborda as dificuldades da utilização do EVM em estudos empíricos.

Este artigo apresenta resultados de um estudo comparativo entre a metodologia teórica e a utilização prática do EVM. Para tanto, foi desenvolvido um estudo de caso múltiplo, com projetos de construção civil de diferentes tipos de obras em empresas públicas e privadas, o que permitiu analisar de forma mais aprofundada os fatores críticos de sucesso, benefícios, dificuldades e limitações da EVM. Dessa forma, este trabalho apresenta uma inovação no tratamento do referido assunto, visto que leva em consideração a análise no âmbito prático e teórico do EVM, tendo como objeto projetos de diferentes características.

\section{Indicador de valor agregado}

\section{Conceitos de valor agregado}

$\mathrm{O}$ indicador de valor agregado avalia $\mathrm{O}$ desempenho do projeto em três dimensões

146 Teixeira Netto, J.; Quelhas, O. L. G.; França, S.; Meirino, M. J. 
fundamentais: o prazo, o escopo e o custo. Essas três dimensões são reconhecidas pelo Project Management Institute (PMI) como o "triângulo de ferro", e é de reconhecida importância seu controle para a eficiência em projetos (PROJECT..., 2013). Para a obtenção dos indicadores de valor agregado é necessário que as atividades sejam quantificadas em custo e que as datas de conclusão de cada tarefa sejam estipuladas. Segundo Vargas (2008), a técnica de valor agregado atende ao objetivo de interligar o custo, o prazo e o escopo.

A análise de valor agregado é também conhecida como Earned Value Management (EVM), ou como Análise de Valor Agregado (AVA). Alguns autores denominam como indicador da curva "S" devido à forma que é representado graficamente, porém neste trabalho será utilizada a nomenclatura EVM.

Segundo Narbaev e De Marco (2014), os principais indicadores de desempenho do EVM são: o Indicador de Desempenho de Custo (Cost Performance Index - CPI) e o Indicador de Desempenho de Prazo (Schedule Performance Index - SPI). Esses indicadores são obtidos a partir de três curvas: Valor Planejado (Planned Value PV), Valor Agregado (EarnedValue - EV) e Custo Real (Actual Cost - AC). As curvas podem ser observadas na Figura 1.

Na Figura 1 pode-se observar o gráfico de valor agregado, também conhecido como curva "S". Os principais indicadores de desempenho do EVM são o Indicador de Desempenho de Custo (CPI Cost Performance Indicator) e o Indicador de Desempenho de Prazo (SPI - Schedule Performance Indicator). Esses indicadores são calculados de acordo com as seguintes fórmulas: $\mathrm{CPI}=\mathrm{EV} / \mathrm{AC}$ e SPI $=\mathrm{EV} / \mathrm{PV}$. Os valores de EV, $\mathrm{AC}$ e PV são obtidos nas respectivas curvas considerando uma data de referência (NARBAEV; DE MARCO, 2014).

O padrão ANSI/EAI 748 (AMERICAN..., 1998) define 32 processos para a obtenção do EVM. Esses processos foram posteriormente simplificados pelo PMBOK, em 1996. Segundo Fleming e Koppelman (2010), essa simplificação trouxe grande aceitação no uso do indicador e facilidade na utilização em diferentes projetos. A simplificação proposta é composta dos seguintes processos principais: planejar todo o trabalho para o projeto, decompor o escopo (EAP), definir os responsáveis, criar um plano de contas integrado à EAP, integrar o escopo com o cronograma e custo, gerar uma linha base, criar uma baseline de controle de mudança, criar um critério claro de medição, avaliar objetivamente o trabalho realizado, analisar as variações do planejamento, avaliar o impacto dos indicadores de previsão e usar o EVM para o gerenciamento dos processos internos da empresa.

\section{Figura 1 - Gráfico de valor agregado}

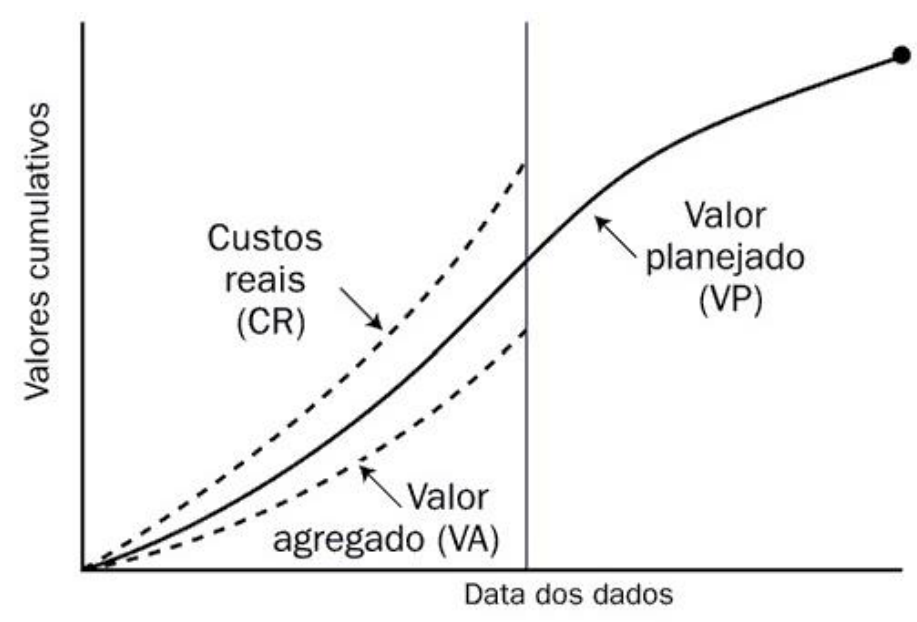

Tempo

Fonte: adaptado de Narbaev e De Marco (2014). 
O indicador de EVM apresenta limitações, descritas na literatura. Alguns autores argumentam que sua aplicação pode incentivar as equipes a realizar tarefas mais fáceis (KIM; BALLARD, 2001). Já outros autores consideram que o Índice de Custo (IDC) apresenta instabilidade durante o andamento do projeto (BUYSE; VANDENBUSSCHE; VANHOUCKE，2009). A pesquisa realizada por diferentes autores recomenda a utilização de indicadores de LPS ${ }^{1}$ (Last Planner System), pois estes apresentariam vantagem em relação ao EVM (KIM; BALLARD, 2010; GRAU et al., 2014). Isso porque no LPS o planejamento e o controle da produção estão divididos em três níveis: o planejamento de longo prazo, o planejamento de médio prazo e o planejamento de curto prazo. No planejamento de curto prazo, a proteção da produção ocorre através da elaboração de planos semanais de produção, sendo incluídas tarefas que tenham suas restrições removidas, incluindo a disponibilidade de recursos (materiais, equipamentos, projeto, entre outros). $\mathrm{O}$ Percentual de Pacotes Concluídos $(\mathrm{PPC})^{2}$ é o principal indicador desse nível de planejamento (BALLARD, 2000; KIM; BALLARD, 2010; GRAU et al., 2014).

Pesquisas sobre o Last Planner System têm sido realizadas no Brasil pelo Núcleo Orientado para a Inovação da Edificação (Norie). Esse núcleo de pesquisa desenvolveu trabalhos sobre a medição do desempenho na construção civil, o que resultou em um sistema de indicadores de qualidade e produtividade para a construção civil denominado Sisind-Net ${ }^{3}$. Esse sistema de indicadores propõe a utilização do indicador PPC em conjunto com os indicadores de valor agregado para uma melhor análise do desempenho de projetos de construção (MOURA; FORMOSO, 2009; COSTA et al. 2005).

\section{Maturidade em valor agregado - EVM3 (Earned Value Maturity Model)}

A maturidade em valor agregado procura identificar a utilização do EVM nas empresas, tema central desta pesquisa. Poucos autores e instituições abordam isso. Entre eles, podem ser citados Stratton (2006), Solomon (2005) e a Nasa (2013).

\footnotetext{
${ }^{1}$ Last Planner System of Production Control. Abordagem de controle de produção baseada naprodução enxuta (MOURA; FORMOSO, 2009).

${ }^{2}$ PPC - Percentual de Pacotes Concluídos. Indicador calculado pela relação da quantidade de pacotes concluídos e o número total de pacotes programados (COSTA et al., 2005).

${ }^{3}$ Sisind-Net. Sistema de Indicadores para Benchmarking na Construção Civil (COSTA; FORMOSO, 2011).
}

Stratton (2006) aborda a utilização do EVM nas empresas através de um modelo de maturidade, que foi desenvolvido por ele com a designação EVM3 (Earned Value Maturity Model), e considera que as empresas podem atingir até cinco níveis de maturidade. Os níveis de maturidade podem ser vistos no Quadro 1.

Segundo Stratton (2006), as categorias do padrão ANSI/EIA 748 são relacionadas aos níveis de maturidade, conforme demonstra o Quadro 2.

Pode ser observado no Quadro 2 que as categorias do padrão ANSI/EIA 748 são utilizadas de maneira crescente, de acordo com o nível de maturidade da empresa em EVM, sendo o padrão utilizado de maneira completa apenas no nível 5 de maturidade.

\section{Fatores críticos de sucesso da utilização do valor agregado}

$\mathrm{Na}$ literatura foram verificadas diferentes abordagens sobre os fatores críticos de sucesso para a implantação do indicador de valor agregado. Em pesquisa do tipo Survey realizada por Kim, Wells Junior e Duffey (2003), com 275 respondentes do PMI, foram identificados diversos fatores críticos de sucesso. Os principais foram: o suporte dos executivos da empresa, o treinamento em EVM, a cultura da organização em EVM e a maturidade da organização em gestão de projetos. Song (2010) utilizou os mesmos fatores críticos de sucesso da pesquisa realizada por Kim, Wells Junior e Duffey (2003), com 677 respondentes do PMI, entre 2007 e 2008, e confirma o treinamento e a prática do EVM como um fator crítico de sucesso. Fleming e Koppelman (2010) argumentam que, para a utilização da análise de valor agregado, é necessário que a empresa tenha maturidade em gerenciamento de projetos.

Lipke (2013) argumenta que, apesar de o indicador de valor agregado ter sido utilizado nos últimos 40 anos, sua utilização não é simples e necessita de um gerenciamento complexo, que envolve o controle de cronograma, o controle preciso de custos e o controle de valor agregado (trabalho realizado). $\mathrm{O}$ autor argumenta que, pela classificação de maturidade em gestão de projetos pela Organizational Project Management Maturity Model (OPM3), a utilização de indicadores se inicia no nível de maturidade 2 (controlado) e evolui até os níveis superiores.

148 Teixeira Netto, J.; Quelhas, O. L. G.; França, S.; Meirino, M. J. 


\section{Quadro 1 - Modelo de maturidade em EVM}

\begin{tabular}{|c|l|l|}
\hline Nível & \multicolumn{1}{|c|}{ Definição EVM3 } & \multicolumn{1}{c|}{ Descrição } \\
\hline $1-\quad$ Ad hoc & $\begin{array}{l}\text { A organização tem pouca ou } \\
\text { nenhuma utilização do EVM. }\end{array}$ & $\begin{array}{l}\text { A organização utiliza o EVM em alguns } \\
\text { projetos piloto. O uso é esporádico e } \\
\text { limitado a alguns projetos. }\end{array}$ \\
\hline 2- Repetitivo & $\begin{array}{l}\text { A organização monitora o EVM } \\
\text { com um sistema de baixo custo, } \\
\text { monitorando o custo total do } \\
\text { projeto e o cronograma. }\end{array}$ & $\begin{array}{l}\text { A organização utiliza o EVM para projetos } \\
\text { grandes ou críticos, indicando que os } \\
\text { processos de EVM podem ser utilizados de } \\
\text { forma repetitiva e com resultados } \\
\text { consistentes. }\end{array}$ \\
\hline 3- Definido & $\begin{array}{l}\text { A organização utiliza o EVM } \\
\text { totalmente de acordo com o } \\
\text { padrão ANSI/EIA 748. }\end{array}$ & $\begin{array}{l}\text { A organização utiliza o EVM como padrão } \\
\text { em toda a empresa para o controle do } \\
\text { projeto. Processos são definidos pela } \\
\text { empresa para obter resultados consistentes. }\end{array}$ \\
\hline 4- Gerenciado & $\begin{array}{l}\text { A organização está fortemente } \\
\text { comprometida com o uso do } \\
\text { EVM, com um sistema para } \\
\text { medir e avaliar o uso do EVM. }\end{array}$ & $\begin{array}{l}\text { A empresa tem um sistema definido para o } \\
\text { EVM com armazenamento do histórico de } \\
\text { projetos, benefícios para projetos futuros e } \\
\text { treinamento regular para a equipe de } \\
\text { projetos. }\end{array}$ \\
\hline 5- Otimizado & $\begin{array}{l}\text { Melhoramento contínuo no uso } \\
\text { do EVM em projetos. }\end{array}$ & $\begin{array}{l}\text { A organização avalia o desempenho da } \\
\text { utilização do EVM de maneira regular e tem } \\
\text { um programa formal de melhoria contínua. }\end{array}$ \\
\hline
\end{tabular}

Fonte: Stratton (2006).

Quadro 2 - Modelo de maturidade em EVM com as categorias da ANSI/EIA 748

\begin{tabular}{|c|c|c|c|c|c|}
\hline \multirow{2}{*}{ Categorias ANSI/EIA 748 } & \multicolumn{5}{|c|}{ Nível de maturidade } \\
\cline { 2 - 6 } & $\mathbf{1}$ & $\mathbf{2}$ & $\mathbf{3}$ & $\mathbf{4}$ & $\mathbf{5}$ \\
\hline Organização & & $\mathrm{X}$ & $\mathrm{X}$ & $\mathrm{X}$ & $\mathrm{X}$ \\
\hline Planejamento, cronograma e orçamentação & & $\mathrm{X}$ & $\mathrm{X}$ & $\mathrm{X}$ & $\mathrm{X}$ \\
\hline Contabilidade & & $\mathrm{X}$ & $\mathrm{X}$ & $\mathrm{X}$ & $\mathrm{X}$ \\
\hline Relatórios e análises & & $\mathrm{X}$ & $\mathrm{X}$ & $\mathrm{X}$ & $\mathrm{X}$ \\
\hline Previsão e manutenção de dados & & $\mathrm{X}$ & $\mathrm{X}$ & $\mathrm{X}$ & $\mathrm{X}$ \\
\hline Métricas de processos & & & & $\mathrm{X}$ & $\mathrm{X}$ \\
\hline Melhoria de processos & & & & & $\mathrm{X}$ \\
\hline
\end{tabular}

Fonte: Stratton (2006).

Vargas (2003), em pesquisa realizada em três obras em empresa da construção civil, identificou os seguintes fatores críticos: treinamento da equipe de planejamento, definição de escopo e suporte gerencial. Valle e Soares (2006), através de um estudo de caso de uma obra de construção civil, identificou fatores críticos de sucesso: o apoio da alta administração, o treinamento da equipe em EVM, o escopo bem definido através da divisão em itens de EAP, o cronograma e o orçamento de acordo com a EAP, a atribuição de responsabilidades a cada item da EAP, o fluxo de trabalho e atividades, o controle de cronograma e custo, o relatório dos indicadores, os procedimentos para análise de consistência, as lições aprendidas e o processo contínuo de aprendizagem.
Buyse, Vandenbussche e Vanhoucke (2009), em estudo de caso em quatro empresas construtoras na Bélgica, identificaram fatores externos e internos para implementar o indicador de valor agregado na construção civil. Os autores citam as seguintes dificuldades: o ceticismo, o desconhecimento do controle completo em custos, o esforço administrativo, o desconhecimento da terminologia e o uso em projetos de longos períodos.

Costa e Formoso (2011) identificaram a necessidade de maturidade em gestão de indicadores para sua utilização. Stratton (2006) e Solomon (2005) demonstraram a necessidade de maturidade em EVM de acordo com os critérios do EVM3. Apesar da diferença metodológica entre estes autores, ambos consideram a maturidade em indicadores um fator crítico de sucesso para sua utilização. 


\section{Método de pesquisa}

A metodologia utilizada na pesquisa é qualitativa e do tipo descritiva, tendo como objeto de estudo a utilização do indicador de valor agregado (EVM) na indústria da construção civil.

A sequência metodológica proposta na pesquisa se encontra na Figura 2.

As etapas da sequência metodológica da pesquisa se encontram descritas a seguir, de acordo com os itens definidos na Figura 2:

(a) Etapa 1: levantamento bibliográfico e revisão da literatura sobre os conceitos do EVM;

(b) Etapa 2: identificação das lacunas na utilização da metodologia do EVM;

(c) Etapa 3: formulação das hipóteses da pesquisa, variáveis, questionário e fontes de evidência da pesquisa;

(d) Etapa 4: estudo de caso piloto (empresa A);

(e) Etapa 5: estudo multicaso nas empresas B, C, $\mathrm{D}$ e E;

(f) Etapa 6: análise dos resultados encontrados nas empresas que participaram do estudo multicaso;

(g) Etapa 7: triangulação e discussão dos resultados obtidos através da comparação entre os resultados encontrados nos projetos do estudo multicaso e na literatura. Segundo Vergara (2006), a triangulação dos resultados tem por objetivo aumentar a validade da pesquisa; $\mathrm{e}$ (h) Etapa 8: conclusão dos resultados encontrados na pesquisa.

$\mathrm{Na}$ Figura 3 encontram-se as hipóteses da pesquisa, que estão descritas nos seguintes tópicos:

(a) H1: as empresas de construção civil não utilizam o modelo teórico do EVM;

(b) a hipótese procura confirmar a pergunta da pesquisa, identificando onde o modelo teórico não é utilizado, por que as empresas não utilizam totalmente a metodologia de valor agregado e em que fase do EVM o modelo não é utilizado;

(c) H2: o modelo teórico de EVM é de difícil utilização;

(d) pretende-se com esta hipótese verificar se os modelos analíticos propostos são complexos e de difícil operacionalização. Entende-se por dificuldade de operacionalização a efetiva implantação dos modelos teóricos nas empresas;

(e) H3: a utilização do EVM cria valor (benefícios) para o projeto;

(f) pretende-se com esta hipótese verificar se a utilização do valor agregado cria valor para a empresa e se há na empresa uma percepção da importância da utilização da análise de valor agregado para o desempenho do projeto;

(g) H4: existem fatores críticos para o sucesso no uso do EVM; e

(h) pretende-se com esta hipótese identificar os principais fatores críticos para o sucesso no uso do EVM.

Figura 2 - Sequência metodológica da pesquisa

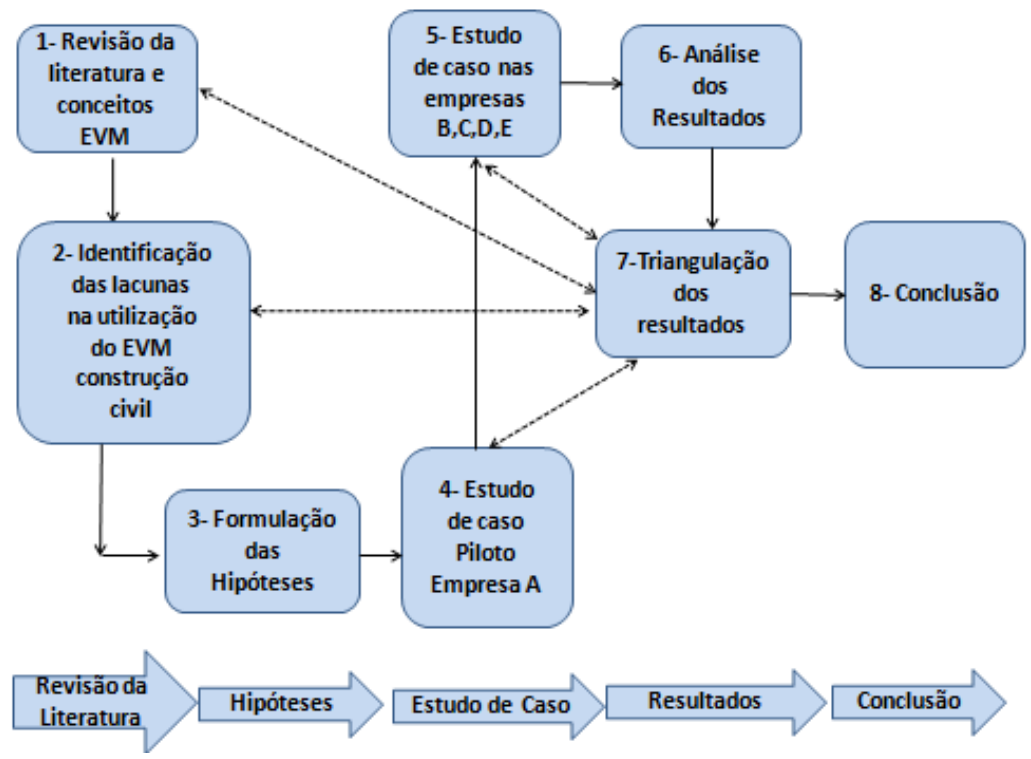

150 Teixeira Netto, J.; Quelhas, O. L. G.; França, S.; Meirino, M. J. 
Figura 3 - Sequência das hipóteses

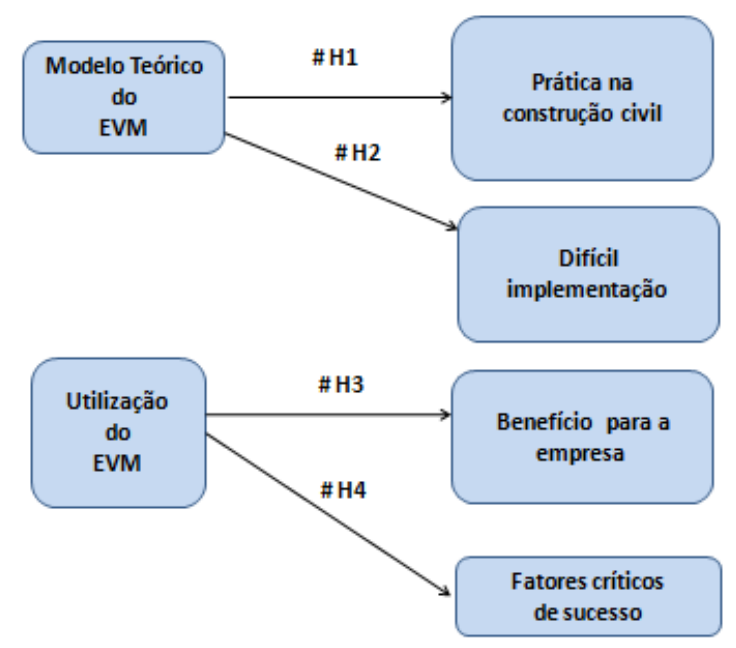

Após a construção das hipóteses, definiu-se um conjunto de variáveis para cada uma. Segundo Bacharach (1989), variáveis são unidades observadas, enquanto os constructos são unidades aproximadas que, por sua simples natureza, não podem ser observadas diretamente.

No Quadro 3 encontram-se os constructos, as variáveis que foram definidas com o objetivo de responder à questão da pesquisa, as fontes de evidência e as referências bibliográficas utilizadas. As variáveis da pesquisa tiveram como base as referências bibliográficas de Marshall (2007), para a hipótese 1 (H1), enquanto nas demais hipóteses foram consideradas as pesquisas de Song (2010) e Kim, Wells Junior e Duffey (2003). As fontes de evidência documentais analisadas foram os relatórios de acompanhamento do projeto, as planilhas de medição, os cronogramas, o acompanhamento físico-financeiro e os procedimentos internos. As entrevistas foram do tipo semiestruturado e conduzidas tomando-se por base o questionário que se encontra no apêndice deste artigo.

Em relação à amostra, Yin (2001) argumenta a necessidade da escolha de casos extremos ou típicos, com o objetivo de ter um resultado revelador. Em relação aos tipos escolhidos da amostra, foi utilizada como referênciaa pesquisa conduzida por Song (2010), que identificou as diferenças entre as empresas privadas $\mathrm{e}$ as públicas. Já a pesquisa de Buyse, Vandenbussche e Vanhoucke (2009) constatou as diferenças em contratos de diferentes portes; e a pesquisa de Stratton (2006) diferenciou as empresas por nível de maturidade em EVM. A partir da utilização das diretrizes mencionadas pelos autores, foram selecionados cinco projetos de construção de diversos portes (pequeno, até $\mathrm{R} \$ 1$ milhão; médio, de R \$ 1 milhão até R \$ 50 milhões; e grande, maior que R\$ 50 milhões), com diferentes tamanhos de empresas (pequena, de 20 a 100 funcionários; média, de 101 a 500 funcionários; e grande, com mais de 500 funcionários), diferentes tipos de estrutura (pública e privada) e diferentes níveis de maturidade em EVM (baixo, médio e alto), sendo realizada a pesquisa em empresas contratadas e contratantes.

\section{Estudo de multicasos: práticas de EVM na construção civil}

Os estudos de casos foram conduzidos, em cada empresa, mediante a utilização do questionário e com as variáveis da pesquisa apresentadas no Quadro 3. A entrevista foi face a face, e o critério de escolha dos entrevistados foi o de exercerem atividades de gestão de projetos, planejamento e controle nas obras selecionadas. Foram entrevistados engenheiros e técnicos nas empresas contratantes e nas construtoras contratadas para a obra. Além disso, foram realizadas análise documental e observações em campo e nos escritórios das empresas selecionadas. Os estudos de casos localizaram-se no Estado do Rio de Janeiro e foram executados entre setembro de 2012 e dezembro de 2013. O Quadro 4 sintetiza os casos analisados. 
Quadro 3 - Conjunto de constructos e variáveis da pesquisa

\begin{tabular}{|c|c|c|c|}
\hline Constructos & Item & Variáveis & Fontes de evidência \\
\hline \multirow{8}{*}{$\begin{array}{l}\text { As empresas de } \\
\text { construção civil } \\
\text { não utilizam o } \\
\text { modelo teórico } \\
\text { do EVM }\end{array}$} & H1.1 & Planejar todo o trabalho para o projeto. & Análise documental \\
\hline & $\mathrm{H} 1.2$ & $\begin{array}{l}\text { Decompor o escopo (EAP) e definir } \\
\text { responsáveis. }\end{array}$ & Análise documental \\
\hline & $\mathrm{H} 1.3$ & Criar um plano de contas integrado à EAP. & Análise documental \\
\hline & H1.4 & $\begin{array}{l}\text { Integrar o escopo com o cronograma e custo, e } \\
\text { gerar uma linha base. Criar uma baseline de } \\
\text { controle de mudança. }\end{array}$ & Análise documental \\
\hline & $\mathrm{H} 1.5$ & Criar um critério claro de medição. & Análise documental \\
\hline & H1.6 & Avaliar objetivamente o trabalho realizado. & Análise documental \\
\hline & H1.7 & $\begin{array}{l}\text { Analisar as variações do planejamento, } \\
\text { impacto dos indicadores de previsão e datas } \\
\text { em que o trabalho deverá ser realizado. }\end{array}$ & $\begin{array}{l}\text { Análise documental e } \\
\text { entrevista }\end{array}$ \\
\hline & $\mathrm{H} 1.8$ & $\begin{array}{l}\text { Usar o EVM para o gerenciamento dos } \\
\text { processos internos da empresa. }\end{array}$ & $\begin{array}{l}\text { Análise documental e } \\
\text { entrevista }\end{array}$ \\
\hline \multirow{3}{*}{$\begin{array}{c}\mathrm{H} 2 \\
\text { O modelo } \\
\text { teórico é difícil } \\
\text { de ser } \\
\text { implementado }\end{array}$} & $\mathrm{H} 2.1$ & $\begin{array}{l}\text { Dificuldade em uma etapa do processo } \\
\text { teórico. }\end{array}$ & $\begin{array}{l}\text { Observações do } \\
\text { pesquisador }\end{array}$ \\
\hline & $\mathrm{H} 2.2$ & Dificuldade na coleta dos dados. & $\begin{array}{l}\text { Observações do } \\
\text { pesquisador }\end{array}$ \\
\hline & $\mathrm{H} 2.3$ & Dificuldade devido a um software adequado. & $\begin{array}{l}\text { Observações do } \\
\text { pesquisador e } \\
\text { entrevista }\end{array}$ \\
\hline \multirow{5}{*}{$\begin{array}{c}\mathrm{H} 3 \\
\text { O valor } \\
\text { agregado cria } \\
\text { valor para o } \\
\text { projeto }\end{array}$} & H3.1 & EVM auxilia na definição do escopo. & Entrevista \\
\hline & $\mathrm{H} 3.2$ & $\begin{array}{l}\text { EVM melhora a comunicação do desempenho } \\
\text { do projeto. }\end{array}$ & Entrevista \\
\hline & $\mathrm{H} 3.3$ & $\begin{array}{l}\text { EVM auxilia o time de projeto para alcançar } \\
\text { os objetivos do cronograma. }\end{array}$ & Entrevista \\
\hline & H3.4 & $\begin{array}{l}\text { EVM auxilia o time de projeto para alcançar } \\
\text { os objetivos do custo do projeto. }\end{array}$ & Entrevista \\
\hline & H3.5 & $\begin{array}{l}\text { EVM auxilia na verificação do serviço } \\
\text { realizado. }\end{array}$ & Entrevista \\
\hline \multirow{8}{*}{$\begin{array}{l}\text { Fatores críticos } \\
\text { de sucesso na } \\
\text { implementação } \\
\text { do EVM }\end{array}$} & $\mathrm{H} 4.1$ & Suporte da alta administração. & $\begin{array}{l}\text { Entrevista e } \\
\text { observações do } \\
\text { pesquisador }\end{array}$ \\
\hline & $\mathrm{H} 4.2$ & Treinamento em EVM. & Entrevista \\
\hline & $\mathrm{H} 4.3$ & $\begin{array}{l}\text { Maturidade da organização em projetos e } \\
\text { EVM. }\end{array}$ & $\begin{array}{l}\text { Observações do } \\
\text { pesquisador }\end{array}$ \\
\hline & $\mathrm{H} 4.4$ & Uso de software integrado de gestão. & Entrevista \\
\hline & $\mathrm{H} 4.5$ & $\begin{array}{l}\text { Cultura da organização e dos executivos } \\
\text { seniores. }\end{array}$ & $\begin{array}{l}\text { Observações do } \\
\text { pesquisador }\end{array}$ \\
\hline & $\mathrm{H} 4.6$ & Experiência do time de projeto em EVM. & Entrevista \\
\hline & $\mathrm{H} 4.7$ & $\begin{array}{l}\text { Adoção de procedimentos padronizados de } \\
\text { EVM na empresa. }\end{array}$ & Entrevista \\
\hline & $\mathrm{H} 4.8$ & Maturidade da organização em EVM. & $\begin{array}{l}\text { Observações do } \\
\text { pesquisador }\end{array}$ \\
\hline
\end{tabular}

Fonte: adaptado de Marshall (2007), Song (2010) e Kim, Wells e Duffey (2003). 
Quadro 4 - Síntese descritiva da amostra

\begin{tabular}{|c|c|c|c|c|}
\hline Projeto & Tipo de obra & Tamanho do projeto & Tamanho da empresa & Tipo de empresa \\
\hline A & $\begin{array}{c}\text { Residencial } \\
\text { unifamiliar }\end{array}$ & R\$ 194 mil & Pequena & Privada \\
\hline B & Estradas & R\$ 190 milhões & Grande & Estatal \\
\hline C & Aeroportuária & R \$ 64 milhões & Grande & Estatal \\
\hline D & Prédio comercial & R \$ 45 milhões & Grande & Autarquia \\
\hline E & $\begin{array}{c}\text { Prédio } \\
\text { residencial }\end{array}$ & R\$ 16 milhões & Média & Privada \\
\hline
\end{tabular}

Um resumo de cada projeto onde foi realizado o estudo de caso se encontra descrito nos itens a seguir.

\section{Projeto A}

A empresa A é uma construtora privada de pequeno porte que iniciou a prática de gerenciamento de projetos há aproximadamente dois anos e utiliza os softwares MsExcel $^{\circledR}$ e MsProject ${ }^{\circledR}$ para o controle do EVM. O projeto é de construção de uma casa de classe média baixa, localizada em um condomínio no município do Rio de Janeiro. Esta tem padrão simples, com aproximadamente $80 \mathrm{~m}^{2}$ de área construída e orçamento de R\$194.000,00. A contratação da obra foi por preço global, e o projeto foi iniciado em setembro de 2012, com prazo previsto de 7 meses. A empresa atua em todo o Estado do Rio de Janeiro, e o estudo de caso foi realizado na empresa construtora.

Quanto à motivação para o uso do EVM foi verificado que a utilização se iniciou devido a recentes solicitações do agente financiador. Além disso, constatou-se que a empresa possui pouca experiência na utilização do EVM e em gestão de projetos.

\section{Projeto B}

A construtora do projeto B é uma grande empresa de construção pesada com atuação em todo o território nacional. A empresa iniciou a prática de gerenciamento de projeto há pouco tempo e contratou um profissional experiente para essa obra, utilizando também os softwares MsExcel ${ }^{\circledR}$ e MsProject ${ }^{\circledR}$ para o controle do EVM. A contratante é uma grande empresa pública da área de energia. O projeto é de construção civil pesada e tem como escopo a construção de uma estrada de acesso de equipamentos para um complexo industrial. O projeto foi iniciado em janeiro de 2012, com prazo previsto de 24 meses e orçamento de $\mathrm{R} \$$ 190.000.000,00. A contratação da obra foi por preço global, e o estudo de caso foi desenvolvido na construtora.
A motivação do uso do EVM ocorreu em função do contrato estabelecido. No entanto, a utilização do valor agregado não fazia parte da cultura da construtora. A empresa teve dificuldade inicial no uso dessa metodologia, que foi superada com a contratação de um profissional experiente em gestão de projetos em EVM. Foi verificado que a utilização do indicador de Percentual de Pacotes Concluídos (PPC) serviu como base para as reuniões semanais do projeto.

\section{Projeto C}

A empresa do projeto $\mathrm{C}$ é uma grande empresa pública de infraestrutura aeroportuária. A empresa contratou uma consultoria externa para o gerenciamento de projeto e utilização do EVM. Essa obra é de construção civil pesada e tem como objeto a ampliação da pista de um aeroporto, iniciada em outubro de 2011. A obra tem duração prevista de 24 meses e orçamento de $\mathrm{R} \$$ 64.000.000,00. A contratação foi por preço global, e foram utilizados os softwares MsExcel ${ }^{\circledR}$ e MsProject ${ }^{\circledR}$. A empresa atua em todo o território nacional, e o estudo de caso foi realizado na empresa contratante.

Quanto à motivação para o uso do EVM foi verificado que a empresa buscava aperfeiçoar a gestão e o controle de projetos.

\section{Projeto D}

A empresa contratante do projeto D é uma grande autarquia pública de produção de vacinas. Esse projeto tem por escopo a construção de um prédio administrativo e almoxarifado de sete andares, tendo sido iniciado em abril de 2012, com duração prevista de 23 meses e valor estimado de $\mathrm{R} \$$ 45.000.000,00. A contratação foi por preço global e foram utilizados os softwares MsExcel ${ }^{\circledR}$ e MsProject ${ }^{\circledR}$. A autarquia atua em todo o território nacional, e o estudo de caso foi realizado na empresa contratante.

Quanto à motivação para o uso do EVM, verificou-se que a utilização se iniciou devido à necessidade de maior controle do desempenho do 
projeto, por iniciativa da equipe de gerenciamento da obra. Foi constatado que a autarquia tinha pouca experiência em EVM e não apresentou práticas consolidadas de gerenciamento de projeto implantadas em suas obras.

\section{Projeto E}

A construtora do projeto E é uma empresa privada de construção civil localizada em Niterói, no estado do Rio de Janeiro, que apresenta práticas de gerenciamento de projeto implantadas em obras há 15 anos. A obra analisada foi um prédio residencial de 13 andares, que se iniciou em maio de 2012, com duração prevista de 19 meses, e orçamento de $\mathrm{R} \$ 16.000 .000,00$.

Quanto à motivação para o uso do EVM foi verificado que a utilização se iniciou devido à necessidade de maior controle no projeto, com ênfase em custos. A diretoria da empresa apoia a utilização de valor agregado, pois identifica vantagens no controle de custos e no planejamento financeiro da execução do projeto. $\mathrm{O}$ apoio foi dado por meio de investimento em treinamento e no desenvolvimento de sistemas de TI. Os gerentes seniores da empresa apoiam e mantêm a cultura da utilização do valor agregado.

\section{Resultados}

Os resultados foram avaliados de acordo com as variáveis definidas no Quadro 3. De acordo com esse quadro, procura-se verificar cada hipótese da pesquisa através dos constructos, que são a utilização do modelo teórico, a dificuldade de implementação, os benefícios gerados para a empresa e os fatores críticos para o sucesso do uso do EVM. Serão mencionados os itens específicos de cada variável que foram mais relevantes em cada estudo de caso. Os resultados encontrados estão descritos a seguir para cada projeto analisado.

\section{Projeto A}

As principais lacunas em relação à metodologia do EVM, que foram identificadas no projeto $\mathrm{A}$, foram a ausência de evidências na utilização do EVM no controle de processos na empresa (H1.8), a não definição do plano de contas (H1.3), a ausência de critérios claros de medição (H1.5) e a dificuldade na transformação dos custos unitários definidos na fase de orçamentação para os custos por pacote de trabalho na fase de planejamento, não integrando o cronograma ao custo (H1.4). Em relação aos benefícios gerados, verificou-se que a visualização gráfica auxilia a comunicação do desempenho do projeto (H3.2). Além disso, a utilização de indicadores de desempenho de custo e de prazo possibilitou antever problemas que possam estar ocorrendo no projeto, auxiliando nos objetivos de prazo (H3.3) e de custo (H3.4). No que tange aos fatores críticos de sucesso, o projeto apresentou carência no treinamento dos funcionários (H4.2), ausência de apoio da alta administração (H4.1), falta de experiência da equipe em análise de valor agregado (H4.6), inexistência de procedimentos padronizados para a utilização de EVM (H4.7) e baixa maturidade em projetos e em EVM (H4.3).

\section{Projeto B}

Foram verificadas as seguintes deficiências em relação à metodologia do EVM identificadas no projeto B: a não definição do plano de contas (H1.3) e não terem sido encontradas evidências na utilização do EVM no controle de processos da empresa (H1.8). Em relação aos benefícios gerados, verificou-se que a visualização gráfica das curvas de custo facilitou a comunicação do desempenho do projeto (H3.2), bem como auxiliou na verificação do serviço realizado e no desempenho de custo (H3.4). As lacunas em relação aos fatores críticos de sucesso foram ausência de treinamento (H4.2), falta de apoio da alta administração (H4.1) e falta de experiência da equipe em análise de valor agregado (H4.6). Observou-se um ponto positivo do projeto, que foi a empresa contratante ter procedimentos definidos para o EVM. Embora o acompanhamento do percentual de pacotes de trabalho (PPC) não seja o foco principal desta pesquisa, sua utilização permitiu melhoria na execução da obra, possibilitando, através do acompanhamento semanal do PPC, a antecipação de problemas no projeto.

\section{Projeto C}

Durante o estudo de caso do projeto $\mathrm{C}$ foi verificada a seguinte deficiência em relação à metodologia do EVM: não foram encontradas evidências na utilização do EVM no controle de processos da empresa (H1.8). A principal dificuldade da implementação do modelo teórico foi a transformação dos custos unitários definidos na fase de licitação em custos por pacote de trabalho (H1.4). Em relação aos benefícios gerados, constatou-se que a visualização gráfica das curvas de custo facilitou a comunicação do desempenho do projeto (H3.2) e que a utilização de indicadores de desempenho serviu como base para medidas corretivas para o prazo (H3.3), custo (H3.4) e serviço realizado (H3.5). Nesse projeto foram identificados os seguintes fatores críticos de sucesso: a contratação de equipe externa com 
experiência em análise de valor agregado (H4.6), o apoio da alta administração (H4.1) e maturidade média em projetos e em EVM (H4.3).

\section{Projeto D}

No que concerne ao projeto $\mathrm{D}$, aferiu-se que as principais dificuldades em relação ao modelo teórico foram a não integração do cronograma aos custos do projeto (H1.4), a não definição dos responsáveis por pacotes de trabalho (H1.2), a utilização do valor atual de custos como valor agregado de custos (H1.6), a não definição clara dos critérios de medição (H1.5) e a utilização restrita do EVM ao setor de planejamento da empresa (H1.8). Em relação aos benefícios para a empresa, observou-se que a visualização gráfica das curvas de custo facilitou a comunicação do desempenho do projeto (H3.2) e a utilização de indicadores de desempenho serviu como base para medidas corretivas para o prazo (H3.3). As lacunas em relação aos fatores críticos de sucesso foram a falta de treinamento (H4.2), a ausência de apoio da alta administração (H4.1), a pequena experiência da equipe em análise de valor agregado (H4.6) e a pouca maturidade em projetos e em EVM (H4.3).

\section{Projeto E}

A empresa iniciou a utilização do EVM há 15 anos, através de planilha eletrônica, tendo aprimorado posteriormente os processos de controle (H4.6). Além disso, armazenou um histórico de projetos de obras e de custos de materiais, o que permitiu que, ao iniciar um novo projeto, os orçamentos fossem realizados com maior precisão (H4.3). A empresa também desenvolveu um software ao longo de 10 anos, que integra os setores de compras, tesouraria e engenharia (H4.4). Atualmente, os materiais são solicitados através de planos de conta do projeto e apropriados no custo da obra na chegada. Verificase que a empresa passou por várias fases de maturidade de gerenciamento de EVM e atingiu alta maturidade de acordo com a classificação do EVM3 (H4.8). Portanto, as principais lacunas identificadas nas outras empresas estudadas nesta pesquisa foram superadas por esta, durante vários anos de aperfeiçoamento contínuo, de treinamento (H4.2) e de melhorias dos processos.

O Quadro 5 resume os resultados encontrados em cada projeto.

\section{Discussão dos resultados}

Nesta seção são discutidos os resultados de acordo com as hipóteses definidas na sequência metodológica da pesquisa. Nessa fase da pesquisa foram realizadas as etapas 6 e 7 da metodologia, constituindo-se da discussão, da triangulação dos resultados encontrados no estudo multicaso e da convergência destes com o exposto por diferentes autores. Os resultados obtidos encontram-se resumidos no Quadro 6. 
Quadro 5 - Resumo dos resultados do estudo multicaso

\begin{tabular}{|c|c|c|c|c|c|}
\hline $\begin{array}{l}\text { Constructos } \\
\text { Resultados }\end{array}$ & Projeto A & Projeto B & Projeto C & Projeto D & Projeto E \\
\hline $\begin{array}{c}\text { H1 } \\
\text { As empresas de } \\
\text { construção civil } \\
\text { não utilizam o } \\
\text { modelo teórico do } \\
\text { EVM. }\end{array}$ & $\begin{array}{l}\text { As lacunas identificadas } \\
\text { foram: não foi definido o } \\
\text { plano de contas (H1.3) e } \\
\text { o custo não foi integrado } \\
\text { ao cronograma (H1.4). } \\
\text { Na fase de execução e } \\
\text { controle não foram } \\
\text { definidos claramente os } \\
\text { critérios de medição } \\
\text { (H1.5) e não foi } \\
\text { utilizado o EVM para o } \\
\text { controle de processos na } \\
\text { empresa (H1.8). }\end{array}$ & $\begin{array}{l}\text { As lacunas identificadas } \\
\text { foram: não foi definido } \\
\text { o plano de contas } \\
\text { (H1.3) e não foi } \\
\text { identificada a utilização } \\
\text { do EVM para o controle } \\
\text { de processos na } \\
\text { empresa (H1.8). }\end{array}$ & $\begin{array}{l}\text { As lacunas } \\
\text { identificadas foram: } \\
\text { a transformação dos } \\
\text { custos unitários em } \\
\text { pacotes de trabalho e, } \\
\text { consequentemente, a } \\
\text { integração do } \\
\text { cronograma (H1.4). } \\
\text { Não foram encontradas } \\
\text { evidências na utilização } \\
\text { do EVM no controle de } \\
\text { processos da empresa } \\
\text { (H1.8). } \\
\end{array}$ & $\begin{array}{l}\text { As lacunas } \\
\text { identificadas foram: na } \\
\text { fase de planejamento a } \\
\text { empresa não integrou o } \\
\text { cronograma aos custos } \\
\text { (H1.4), a não definição } \\
\text { dos responsáveis por } \\
\text { pacotes de trabalho } \\
\text { (H1.2), não foram } \\
\text { definidos claramente os } \\
\text { critérios de medição } \\
\text { (H1.5), a empresa } \\
\text { utilizou o valor atual de } \\
\text { custos como valor } \\
\text { agregado de custos } \\
\text { (H1.6) e não foi } \\
\text { utilizado o EVM para o } \\
\text { controle de processos } \\
\text { na empresa (H1.8). }\end{array}$ & $\begin{array}{l}\text { A empresa utilizou } \\
\text { plenamente o } \\
\text { modelo de EVM. } \\
\\
\end{array}$ \\
\hline $\begin{array}{c}\mathrm{H} 2 \\
\text { O modelo teórico é } \\
\text { difícil de ser } \\
\text { implementado pela } \\
\text { empresa. }\end{array}$ & $\begin{array}{l}\text { Verificou-se dificuldade } \\
\text { no modelo teórico } \\
(\mathrm{H} 2.1)\end{array}$ & $\begin{array}{l}\text { Verificou-se dificuldade } \\
\text { no modelo teórico } \\
(\mathrm{H} 2.1) \text {. }\end{array}$ & $\begin{array}{l}\text { Verificou-se } \\
\text { dificuldade no modelo } \\
\text { teórico }(\mathrm{H} 2.1) \text {. }\end{array}$ & $\begin{array}{l}\text { Verificou-se } \\
\text { dificuldade no modelo } \\
\text { teórico }(\mathrm{H} 2.1)\end{array}$ & $\begin{array}{l}\text { A empresa levou } \\
10 \text { anos para } \\
\text { implantar } \\
\text { integralmente a } \\
\text { análise por valor } \\
\text { agregado. }\end{array}$ \\
\hline $\begin{array}{c}\mathrm{H} 3 \\
\text { O EVM cria valor } \\
\text { para o projeto. }\end{array}$ & \begin{tabular}{|l|} 
Foi verificado que sim, \\
pois permitiu o \\
acompanhamento \\
através da visualização \\
gráfica (H3.2), e a \\
utilização de indicadores \\
de desempenho de custo \\
e prazo possibilitou \\
antever problemas, o que \\
auxiliou nos objetivos de \\
prazo (H3.3) e custo \\
(H3.4).
\end{tabular} & $\begin{array}{l}\text { Foi verificado que sim, } \\
\text { pois permitiu o } \\
\text { acompanhamento e a } \\
\text { visualização gráfica das } \\
\text { curvas de custo, } \\
\text { facilitou a comunicação } \\
\text { do projeto (H3.2), bem } \\
\text { como auxiliou na } \\
\text { verificação do serviço } \\
\text { realizado e no } \\
\text { desempenho de custo } \\
\text { (H3.4). }\end{array}$ & $\begin{array}{l}\text { Foi verificado que sim, } \\
\text { pois permitiu o } \\
\text { acompanhamento e a } \\
\text { visualização gráfica das } \\
\text { curvas de custo, } \\
\text { facilitou a comunicação } \\
\text { do projeto (H3.2), bem } \\
\text { como auxiliou na } \\
\text { verificação do serviço } \\
\text { realizado e no } \\
\text { desempenho de custo } \\
(\text { H3.4), prazo (H3.3) e } \\
\text { serviço realizado } \\
\text { (H3.5). }\end{array}$ & $\begin{array}{l}\text { Foi verificado que cria } \\
\text { valor, pois permitiu o } \\
\text { acompanhamento e a } \\
\text { visualização gráfica das } \\
\text { curvas de custo, } \\
\text { facilitou a comunicação } \\
\text { do projeto (H3.2), o } \\
\text { desempenho e } \\
\text { cumprimento do prazo } \\
\text { (H3.3). }\end{array}$ & $\begin{array}{l}\text { Foi identificado } \\
\text { que criou valor } \\
\text { para a empresa nas } \\
\text { etapas de } \\
\text { planejamento, } \\
\text { controle e custos } \\
\text { das obras. }\end{array}$ \\
\hline $\begin{array}{c}\text { H4 } \\
\text { Há fatores críticos } \\
\text { para o sucesso do } \\
\text { uso do EVM. }\end{array}$ & $\begin{array}{l}\text { As lacunas identificadas } \\
\text { foram: falta de } \\
\text { treinamento (H4.2), falta } \\
\text { de apoio da alta } \\
\text { administração (H4.1), } \\
\text { falta de experiência da } \\
\text { equipe (H4.6), } \\
\text { inexistência de } \\
\text { procedimentos } \\
\text { padronizados para a } \\
\text { utilização de EVM } \\
\text { (H4.7) e baixa } \\
\text { maturidade em projetos } \\
\text { e em EVM (H4.3). }\end{array}$ & $\begin{array}{l}\text { A empresa conseguiu } \\
\text { utilizar o EVM devido } \\
\text { ao treinamento da } \\
\text { equipe (H4.2). A } \\
\text { empresa teve apoio da } \\
\text { alta administração } \\
\text { (H4.1) e de experiência } \\
\text { da equipe em valor } \\
\text { agregado (H4.6). }\end{array}$ & $\begin{array}{l}\text { Foi contratada equipe } \\
\text { externa com } \\
\text { experiência em gestão } \\
\text { de projetos e EVM } \\
\text { (H4.6). Houve apoio da } \\
\text { alta administração } \\
(\text { H4.1) e maturidade } \\
\text { média em EVM (H4.3). }\end{array}$ & $\begin{array}{l}\text { As lacunas } \\
\text { identificadas foram: a } \\
\text { falta de treinamento } \\
\text { (H4.2), a falta de apoio } \\
\text { da alta administração } \\
\text { (H4.1), pouca } \\
\text { experiência da equipe } \\
\text { (H4.6) e baixa } \\
\text { maturidade em EVM } \\
\text { (H4.3). }\end{array}$ & $\begin{array}{l}\text { Foi verificado que } \\
\text { a empresa fez } \\
\text { treinamento da } \\
\text { equipe (H4.2) e } \\
\text { desenvolveu um } \\
\text { sistema integrado } \\
\text { de custos e EVM } \\
\text { (H4.4). Houve } \\
\text { busca constante da } \\
\text { empresa em } \\
\text { melhoria nos } \\
\text { processos de EVM } \\
\text { e maturidade alta } \\
\text { em EVM (H4.8). }\end{array}$ \\
\hline
\end{tabular}




\section{Quadro 6 - Comparação teórica dos resultados e referências dos autores}

\begin{tabular}{|c|c|c|}
\hline Constructos & Resultados & Autores \\
\hline $\begin{array}{c}\text { H1 } \\
\text { As empresas de } \\
\text { construção civil } \\
\text { não utilizam o } \\
\text { modelo teórico } \\
\text { do EVM. }\end{array}$ & $\begin{array}{l}\text { Falta de integração do cronograma ao custo do } \\
\text { projeto, não utilização de plano de contas, falta de } \\
\text { critérios claros de medição nos projetos e não } \\
\text { utilização do EVM para o controle de processos } \\
\text { na empresa. }\end{array}$ & $\begin{array}{l}\text { De Marco e Narbaev } \\
\text { (2013), Lipke (2013). }\end{array}$ \\
\hline \multirow{2}{*}{$\begin{array}{c}\mathrm{H} 2 \\
\text { O modelo teórico } \\
\text { é difícil de ser } \\
\text { implementado. }\end{array}$} & $\begin{array}{l}\text { Identificação da dificuldade de implementar o } \\
\text { modelo teórico em sua totalidade. }\end{array}$ & Lipke (2013). \\
\hline & $\begin{array}{l}\text { Dificuldade relativa ao uso de um software } \\
\text { adequado, que possibilite a integração do custo } \\
\text { do projeto ao cronograma. }\end{array}$ & $\begin{array}{l}\text { Kim, Wells Junior e } \\
\text { Duffey (2003). }\end{array}$ \\
\hline $\begin{array}{l}\text { H3 } \\
\text { O valor agregado } \\
\text { cria valor para o } \\
\text { projeto. }\end{array}$ & $\begin{array}{l}\text { Controle do custo e escopo do projeto } \\
\text { proporciona melhor comunicação do desempenho } \\
\text { do projeto através da visualização gráfica dos } \\
\text { indicadores, auxiliando a equipe de projeto a } \\
\text { alcançar os objetivos do cronograma e custos. }\end{array}$ & $\begin{array}{l}\text { Song (2010), Kim, } \\
\text { Wells Junior e } \\
\text { Duffey (2003). }\end{array}$ \\
\hline \begin{tabular}{l}
\multicolumn{1}{c}{$\mathrm{H} 4$} \\
Fatores críticos \\
de sucesso na \\
implementação \\
do EVM.
\end{tabular} & $\begin{array}{l}\text { Apoio da alta administração, treinamento da } \\
\text { equipe em EVM, escopo bem definido através da } \\
\text { divisão em itens de EAP, maturidade em gestão } \\
\text { de projetos e maturidade em EVM3. }\end{array}$ & $\begin{array}{l}\text { Valle (2006), Vargas } \\
\text { (2003), Lipke (2013) } \\
\text { e Stratton (2006). }\end{array}$ \\
\hline
\end{tabular}

No Quadro 6 encontram-se as hipóteses da pesquisa, os respectivos resultados e os autores a eles relacionados. Os resultados da pesquisa para cada hipótese são discutidos a seguir.

(a) H1: as empresas de construção civil não utilizam o modelo teórico do EVM: constatou-se que a maior parte das empresas utilizou o modelo teórico de maneira parcial. Nos projetos A, C e D a principal lacuna foi a ausência da integração do cronograma. Nos projetos A e D foi a falta de critérios claros de medição nos projetos. Nos projetos A, B, C e D não foi utilizado o EVM para o controle de processos na empresa. Observou-se que somente no projeto $\mathrm{E}$ foi utilizado plenamente o modelo teórico de EVM;

(b) Conforme a revisão da literatura realizada, De Marco e Narbaev (2013) identificaram que as empresas da construção civil não utilizam a metodologia de EVM em sua totalidade. Além disso, Lipke (2013) também constatou a necessidade do controle do cronograma e dos custos para a utilização do EVM;

(c) H2: o modelo teórico é difícil de ser implementado: foram verificadas dificuldades relativas ao modelo teórico em relação à integração do cronograma aos custos. Conforme a revisão da literatura realizada, Lipke (2013) identificou a dificuldade de implementar o modelo teórico do EVM em sua totalidade. Observou-se também que a utilização de um software adequado na empresa $\mathrm{E}$ permitiu melhorias na utilização do EVM, auxiliando a integração entre os diferentes setores da empresa. Sob esse mesmo aspecto, Kim, Wells Junior e Duffey (2003) também identificaram essa necessidade;

(d) H3: a utilização do valor agregado cria valor para o projeto: observou-se em todas as empresas analisadas que a utilização do EVM possibilitou maior controle do custo e do escopo do projeto, além de ter proporcionado melhor comunicação do desempenho dele através da visualização gráfica dos indicadores, auxiliando a equipe do projeto a alcançar os objetivos do cronograma e dos custos. A utilização de indicadores de desempenho permitiu antever problemas, servindo de base para medidas corretivas nos projetos. Os resultados encontrados, em relação aos benefícios do uso do valor agregado, coincidem com as pesquisas realizadas por Song (2010) e Kim, Wells Junior e Duffey (2003); e

(e) H4: existem fatores críticos para o sucesso do uso do EVM. Verificaram-se os seguintes aspectos em relação aos fatores críticos de sucesso: o apoio da alta administração, o treinamento da equipe em EVM e o escopo bem definido através da divisão em itens de EAP, fatos esses observados também nas pesquisas de Valle e Soares (2006) e Vargas (2003). Foi reconhecido ainda que as empresas com maior maturidade em gestão de projetos foram as que apresentaram melhor desempenho na utilização do valor agregado, fato que coincide com o observado por Lipke (2013) em sua pesquisa. Adicionalmente aos itens citados, verificou-se que o tipo de escopo da obra de cada 
estudo de caso não é um fator relevante, uma vez que a importância reside na definição clara do escopo e na divisão adequada dos pacotes de trabalho, conforme também constado por Vargas (2003) em sua pesquisa. A abordagem de Stratton (2006) analisa a utilização do valor agregado de acordo com os diferentes níveis de maturidade em EVM. Seguindo essa abordagem, foi confirmado nesta pesquisa que as empresas B, C e E, com maior maturidade em EVM, utilizam com maior abrangência o modelo teórico proposto.

É importante ressaltar que a discussão e a triangulação dos resultados revelaram dificuldades na implementação da totalidade do modelo teórico, além de demonstrarem fatores críticos de sucesso e benefícios para a empresa na utilização do valor agregado em projetos da construção civil. Ao se compararem as diferentes empresas analisadas no estudo multicaso, constatou-se que apenas o projeto E utilizou o EVM em sua totalidade. Isso ocorre em razão de essa empresa ter atingido um nível alto de maturidade em EVM, após ter ultrapassado os níveis em que as demais empresas se encontravam.

\section{Conclusão}

O presente trabalho procurou analisar a gestão de projetos da construção civil utilizando o EVM. A proposta da pesquisa foi analisar as práticas da utilização da gestão de desempenho do valor agregado no âmbito prático através do estudo de caso múltiplo, comparando-os com a fundamentação teórica. Buscou-se ainda identificar os fatores críticos de sucesso, os benefícios e as limitações na utilização do EVM. O método utilizado para identificar as diferenças entre os modelos e as práticas organizacionais da utilização do EVM permitiu a verificação das hipóteses e a conclusão da pesquisa. De acordo com a análise da metodologia utilizada, pode-se afirmar que o estudo multicaso possibilitou obter diferentes visões do uso do indicador de valor agregado, uma vez que foram analisadas empresas privadas $\mathrm{e}$ públicas de diferentes portes e com diferentes formas de contratação.

A principal contribuição desta pesquisa foi verificar que a utilização completa da metodologia de EVM é complexa e envolve um controle preciso de custos, do cronograma e do controle do valor agregado. Em razão disso, observou-se que em algumas empresas estudadas a utilização do modelo teórico de EVM foi realizada de maneira parcial. Sob esse prisma, a maior dificuldade das empresas foi a integração entre o controle de custos de projeto e do cronograma.
Outra contribuição de caráter gerencial para projetos de obras foi a identificação de que a utilização do EVM não deve ser restrita apenas ao setor de planejamento, devendo compreender também o setor financeiro, o setor de compras e a produção em campo. Portanto, esses setores devem trabalhar de forma integrada para que os dados de custo e de prazo do projeto possam ser coletados e sejam consolidados no setor de planejamento. Verificou-se ainda que a utilização de um software adequado possibilitou que os dados fossem coletados e consolidados mais facilmente. Além disso, verificou-se que o EVM pode ser um importante instrumento de apoio à decisão para projetos de construção civil, permitindo que desvios em relação ao planejamento possam ser identificados e corrigidos.

Apesar de não ser o foco principal desta pesquisa, foram encontradas algumas evidências, ainda não conclusivas, de que a utilização do indicador Percentual de Pacotes Concluídos (PPC), juntamente com o EVM, auxiliou no desempenho do projeto, removendo restrições ao andamento da obra no curto prazo. O PPC possibilitou ainda um melhor acompanhamento da produção semanal e propicia que possíveis distorções do planejamento possam ser corrigidas mais rapidamente. No que tange a uma contribuição de caráter acadêmico, este artigo confirma outras pesquisas realizadas por autores de diferentes países sobre o indicador de valor agregado, conforme resumido no Quadro 6.

Por fim, o artigo verificou os fatores críticos para o uso do valor agregado, tais como o treinamento da equipe, a maturidade em gestão de projetos, o apoio da alta administração, a necessidade de procedimentos predefinidos e a utilização de um software adequado, que possa fazer a integração entre o custo e o cronograma do projeto. Observou-se ainda que o EVM propiciou benefícios, possibilitando maior controle do custo e do escopo do projeto, além de ter proporcionado melhor comunicação do desempenho dele através da visualização gráfica dos indicadores, auxiliando a equipe do projeto a alcançar os objetivos do cronograma e dos custos.

\section{Referências}

AMERICAN NATIONAL STANDARDS
INSTITUTE. ELECTRONIC INDUSTRIES
ASSOCIATION. Earned Value Management
Systems: ANSI/EIA-748. Arlington, 1998.
Systems: ANSI/EIA-748. Arlington, 1998.

AL-JIBOURI, S. Monitoring Systems and Their Effectiveness For Project Control in Cnstruction. International Journal of Project Management, v. 21, n. 2, p. 145-154, 2003. 
BALLARD, G. The Last Planner System of Production Control. Birmingham, 2000. 192 f. Tese (Doutorado em Engenharia Civil) - Escola de Engenharia, Universidade de Birmingham, Birmingham, 2000.

BACHARACH, S. B. Organizational Theories: some criteria for evaluation. Academy of Management Review, v. 14, n. 4, p. 406-515, 1989.

BUYSE, P.; VANDENBUSSCHE, T.; VANHOUCKE, M. Performance Analysis of Earned Value Management in the Construction Industry. Gent-Béligica, 2009. 97 p. Dissertação (Mestrado em Economia) - Escola de Economia de Bedrijfskunde, Gent-Béligica, 2009.

COSTA, D. B. et al. Sistema de Indicadores Para Benchmarking na Construção Civil: manual de utilização. Escola de Engenharia Civil, Universidade Federal do Rio Grande do Sul, Porto Alegre, 2005.

COSTA, D. B.; FORMOSO, C. T. Fatores-Chave de Sucesso Para Sistemas de Indicadores de Desempenho Para Benchmarking Colaborativo Entre Empresas Construtoras. Ambiente Construído, Porto Alegre, v. 11, n. 3, p. 143-159, jul./set. 2011

DE MARCO, A.; NARBAEV, T. Earned ValueBased Performance Monitoring of Facility Construction Projects. Journal of Facilities Management, v. 11, n. 1, p. 69-80, 2013.

DOBBS, R. et al. Infrastructure Productivity: how to save \$1 trillion a year. Mckinsey Global Institute, jan. 2013. Disponível em: <http://www.mckinsey.com/insights/ engineering_construction/infrastructure_productivi ty>. Acesso em: 28 nov. 2014.

FLEMING, Q. W.; KOPPELMAN, J. M. Earned Value: project management. 4. ed. Pennsylvania, PMI, 2010.

GRAU, D. et al. A Combined Planning and Controls Approach to Accurately Estimate, Monitor, and Stabilize Work Flow. In: COMPUTING IN CIVIL AND BUILDING ENGINEERING, Washington, 2014.

Proceedings... Washington: ASCE, 2014.

KIM, E. H.; WELLS JUNIOR, W. G.; DUFFEY, M. R. A Model For Effective Implementation of Earned Value Management Methodology. International Journal of Project Management, v. 21, n. 5, p. 375-382, 2003.

KIM, Y.-W.; BALLARD, G. Earned Value Method and Customer Earned Value. Journal of Construction Research, v. 3, n. 1, p. 55-66, 2001.
KIM, Y.-W.; BALLARD, G. Management Thinking in the Earned Value Method System and the Last Planner System. Journal of Management in Engineering, v. 26, n. 4, p. 223-228, 2010.

LIPKE, W. Is Something Missing From Project Management? Cross Talk, 2013. p. 17. Disponível em: <http://www.crosstalkonline.org/ storage/issue-archives/ 2013/ 201307/201307Lipke.pdf>. Acesso em: 10 jun. 2014.

MARSHALL, R. A. The Contribution of Earned Value Management to Project Success on Contracted Efforts. Journal of Contract Management, p. 21-33, summer, 2007.

MOURA, C. B.; FORMOSO, C. T. Análise Quantitativa De Indicadores de Planejamento e Controle da Produção: impactos do Sistema Last Planner e fatores que afetam a sua eficácia. Ambiente Construído, Porto Alegre, v. 9, n. 3, p. 57-74, jul./set. 2009.

NARBAEV, T.; DE MARCO, A. Combination of Growth Model and Earned Schedule to Forecast Project Cost at Completion. Journal of Construction Engineering and Management, v. 140, n. 1, 2014.

NASA, Earned Value Management (EVM) Implementation Handbook. National Aeronautics and Space Administration, February 2013. Disponível em: <http://evm.nasa.gov/docs/Handbooks/EVM_Impl ementation_Handbook_STI13-059.pdf>. Acesso em: 26 set. 2014.

\section{PROJECT MANAGEMENT BODY OF} KNOWLEDGE. Um Guia do Conhecimento de Gerenciamento em Projetos: guia PMBOK. 5. ed. Pennsylvania: PMI Publishing Division, 2013.

PRACTICE STANDARD FOR EARNED VALUE MANAGEMENT: Standard EVM 2nd ed. Newtown Square, Pennsylvania: Pmi Publishing Division, 2011.

SOLOMON, P. J. Performance-Based Earned Value $^{\circledR}$. In: ANNUAL INTERNATIONAL SYMPOSIUM OF THE INTERNATIONAL COUNCIL ON SYSTEMS ENGINEERINGINCOSE, 15., Seatle, 2005. Proceedings... Seatle, 2005.

SONG, L. Earned Value Management: a global and cross-industry perspective on current EVM practice. Project Management Institute, 2010.

STRATTON, R. W. The Earned Value Management Maturity Model. Management Concepts Inc., 2006. 
VALLE, J. A.; SOARES, C. A. P. The Use of Earned Value Analysis (EVA) in the Cost Management of Construction Projects.In: PMI GLOBAL CONGRESS EMEA, Pennsylvania, 2006. Proceedings... Pennsylvania, 2006.

VANHOUCKE, M. Measuring the Efficiency of Project Control Using Fictitious and Empirical Project Data. International Journal of Project Management, v. 30, n. 2, p. 252-263, 2012.

VARGAS, R. V. Análise de Valor Agregado em Projetos. 3. ed. Rio de Janeiro: Brasport, 2008.
VARGAS, R. V. Earned Value Analysis in the Control of Projects: success or failure? AACE International Transactions, v. 21, n. 4, p. 211214, 2003.

\section{VERGARA, S. C. Métodos de Pesquisa em} Administração. São Paulo: Atlas, 2006.

YIN, R. Estudo de Caso: planejamento e métodos. 2. ed. Porto Alegre: Bookman, 2001.

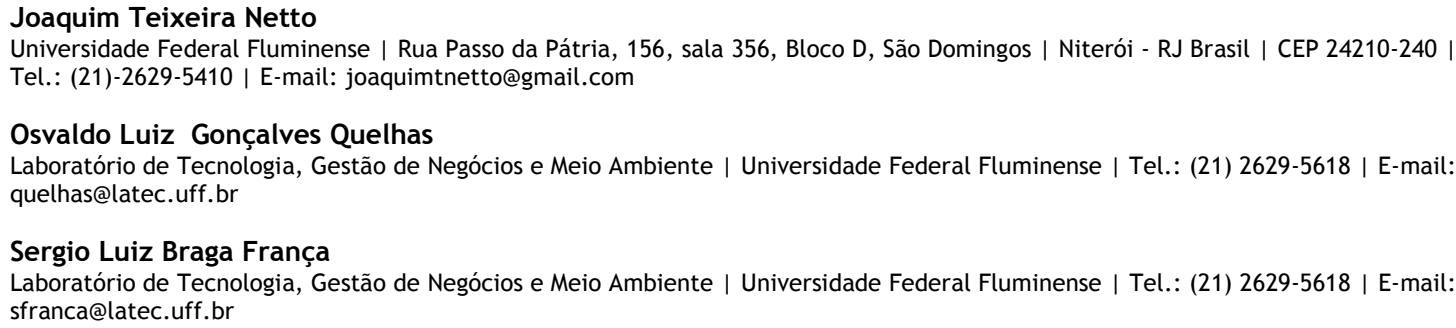

Marcelo Jasmim Meirino

Laboratório de Tecnologia, Gestão de Negócios e Meio Ambiente | Universidade Federal Fluminense | Tel.: (21) 2629-5618 | E-mail: marcelo@latec.uff.br

Revista Ambiente Construído

Associação Nacional de Tecnologia do Ambiente Construído

Av. Osvaldo Aranha, $99-3^{\circ}$ andar, Centro

Porto Alegre - RS - Brasil

CEP $90035-190$

Telefone: +55 (51) 3308-4084

Fax: +55 (51) 3308-4054

www.seer.ufrgs.br/ambienteconstruido

E-mail: ambienteconstruido@ufrgs.br 